SUSX-TH-98-018, hep-ph/9807372

\title{
Constraints on Axion Models
}

\author{
Mark Hindmarsh* and Photis Moulatsiotis ${ }^{\dagger}$ a \\ ${ }^{a}$ Centre for Theoretical Physics \\ University of Sussex \\ Brighton BN1 9QJ \\ U.K
}

Two particular classes of axion models are presented, each one yielding a lower bound on the axion decay constant, based though on different considerations. In the first class only some, and not all, of the right-handed quarks have PQ charges, whereas in the second one the left-handed sector of the same quarks is taken into account as well. In the first case we find that bounds coming from astrophysics are significantly relaxed compared with those for the DFSZ. As for the second class, the astrophysical constraints proved to be less severe (with one exception), than those coming from FCNC processes.

\section{INTRODUCTION}

The axion [1,2] is a particle which inevitably appears in one of the most appealing ways of solving the strong CP problem, which is the hypothesis that there is an extra global U(1) symmetry of the fermions of the Standard Model, known as the Peccei-Quinn (PQ) symmetry. The current benchmarks for realistic axion theories are the KSVZ [3] and DFSZ [4] models, to which most experiments are compared. However, there are in principle many models, distinguished by different assignments of the quark and lepton transformation properties under the Peccei-Quinn symmetry. This was realised, soon after the original axion model was proposed, by two groups [5.6]. However, all axion models based on identifying the electroweak scale with the scale of PQ symmetry-breaking were quickly ruled out by accelerator experiments and more seriously by astrophysical arguments based on energy loss from stars and supernovae (see [7] for reviews). The DFSZ and KSVZ models are examples of "invisible" axion models, which break the PQ symmetry with a singlet Higgs field. The supervnova constraints [8,9] bound the PQ scale to be above

\footnotetext{
*m.b.hindmarsh@sussex.ac.uk

†p.moulatsiotis@sussex.ac.uk
}

about $10^{10} \mathrm{GeV}$. It took a few years for the models of Peccei, Wu and Yanagida, and of Krauss and Wilczek, to be extended with a Higgs singlet 10,11, to be named "variant" axion models.

These models assign different $\mathrm{PQ}$ charges to different right-handed quarks: in the DFSZ model all quarks of a given chirality have the same charge. Altering only the right-handed sector avoids any potential difficulties with flavourchanging neutral currents (FCNCs). The question of motivation arises: why would we want to do this in the first place? There are three reasons: firstly, models with only one PQ-charged righthanded quark do not have an axion domain wall problem [12]; secondly, one can use a PQ symmetry to distinguish the top quark and potentially explain its relatively large mass; and thirdly, one should explore all possibilities for axion models given the importance of the PQ solution of the strong CP problem.

The cosmological domain wall problem arises in theories in which the Universe undergoes a phase transition at which the PQ symmetry is broken. Axion strings form 12,13, which subsquently get connected by $2 A$ domain walls, where $A$ is the QCD anomaly factor, which is either a halfinteger or an integer. Only in the case $A=1 / 2$ can the Universe avoid being subsequently domi- 
nated in energy density by the resulting network of walls and strings. When $A=1 / 2$ each string is connected to one wall only, and can be drawn under the wall's surface tension to a neighbouring piece of anti-string, and annihilate. We shall see that some of our models have $A=1 / 2$, and thus represent the simplest way so far of constructing viable models in the absence of inflation.

In the first part of this work, we shall investigate the supernova constraints on variant axion models, which are significantly different from those on the DFSZ model [14]. In the second part, in the spirit of inquiry, we consider the effect of different charges on the left-handed quarks. Here we must tackle full on the question of FCNCs, which constrain both the PQ scale, through the decays $K^{+} \rightarrow \pi^{+} a$, and the masses of the other pseudoscalars in the theory, through the constraints on $B \bar{B}$ mixing. In doing so we are greatly aided by the recent work of Feng et al. [15], who comprehensively considered FCNC constraints on models with family symmetry. Our axion models are particular cases with an anomalous abelian symmetry.

There are many types of model, depending on how the PQ charges are assigned 16,17]. In this work we examine a class of models with very strong constraints on the axion scale, stronger even than the supernova constraints with one exception, the case where only the top quark has a PQ charge. The constraint on $B \bar{B}$ mixing requires that the other pseudoscalars should generically have masses greater than about $10^{6} \mathrm{GeV}$. While not enough to rule out the models, it certainly renders them unattractive, as one has to prevent this mass scale from leaking into the Standard Model sector via radiative corrections.

\section{VARIANT AXION MODELS}

Variant axion models have two Higgs doublets $\phi_{1}$ and $\phi_{2}$ and one Higgs singlet $\phi$, whose vacuum expectation values are

$$
\begin{array}{r}
\left\langle\phi_{1}\right\rangle=\frac{1}{\sqrt{2}}\left(\begin{array}{c}
v_{1} \\
0
\end{array}\right), \quad\left\langle\phi_{2}\right\rangle=\frac{1}{\sqrt{2}}\left(\begin{array}{c}
0 \\
v_{2}
\end{array}\right), \\
\langle\phi\rangle=\frac{v}{\sqrt{2}} .
\end{array}
$$

In the DFSZ model, $\phi_{1}$ is used to give a mass to $u$-type quarks and $\phi_{2}$ to the $d$-type quarks, with both having the same PQ charge, which can be normalised to \pm 1 , with the singlet having $\mathrm{PQ}$ charge $1 / 2$. In the variant models, only one of the Higgs fields has a PQ charge (which we can choose to be $\phi_{1}$ ), and this field is responsible for the masses of the quarks with PQ charges. The models are thus distinguished by which of the righthanded quarks is charged. In Table 1 we list the models (including three extra ones not considered in (14]). We cannot be any freer with the quark

Table 1

The quarks with Peccei-Quinn charge 1 in the variant axion models considered in the text. The rest of the quarks (and leptons) have PQ charge zero.

\begin{tabular}{ll}
\hline Model & PQ-charged quark(s) \\
\hline I & $u_{R}$ \\
II & $t_{R}$ \\
III & $u_{R}, t_{R}$ \\
IV & $c_{R}$ \\
V & $c_{R}, u_{R}$ \\
VI & $c_{R}, t_{R}$ \\
\hline
\end{tabular}

charge assigments without complicating the Higgs structure further. Note that $\phi_{2}$ gives masses to both the $u$-type and $d$-type PQ-neutral fermions, just as in the Standard Model Higgs.

The QCD anomaly factor $A$ is given by

$A=\sum_{q}\left(Q_{R q}-Q_{L q}\right) t_{q}$,

where $q$ labels a chiral quark state in a representation with quadratic Casimir $t_{q}$. For $\mathrm{SU}(3)$ triplets, $t_{q}=1 / 2$, and thus $A=1 / 2$ for models I, II and IV. These are the models with no domain wall problem.

We define the axion scale $v_{a}$ through the kinetic term in the Lagrangian for the axion under the space-time dependent transformation $\exp (i \alpha Q)$, where $Q$ is the generator of the PQ charge $\mathcal{L}_{K}=$ $\frac{1}{2} v_{a}^{2}(\partial \alpha)^{2}$. Note that many authors define an axion scale $f_{a}$, which is related to our $v_{a}$ by 
$f_{a}=v_{a} / 2 A$. The advantage of this definition is that the mass of the axion, and its coupling to gauge bosons, are inversely proportional to $f_{a}$.

The supernova constraint ultimately arises from nucleon bremsstrahlung in the nascent neutron star [3]: if the axion couplings to the nucleons is too strong, the resulting axion flux will change the picture of the supernova explosion, which is accurate enough to give reasonably good neutrino fluxes. The limit [9] on the couplings $h_{a n}$ and $h_{a p}$, the pseudoscalar couplings to the neutron and proton respectively, is quoted as

$\left(2 h_{\text {an }}^{2}+h_{\text {ap }}^{2}\right)^{1 / 2}<2.85 \times 10^{-10}$,

which is a fit to the boundary of a numerically determined excluded region. There are many uncertainties in the calculation, and we have taken a conservative value from [9] which included possible many-body effects on the axion production rate. Thermal pion conversion $\pi N \rightarrow N^{\prime} a$ has been ignored, as it is thought to be unimportant.

The calculation of the couplings $h_{a n}$ and $h_{a p}$ can be found in 14 for Models I to III, and can be straightforwardly extended to Models IV to VI. We do not reproduce them here, and instead merely exhibit the limit on $v_{a}$ which results from the relation $h_{a N} \sim m_{N} / v_{a}$ (Figure 1). The constraint on Model I, where the $u$ quark only is singled out, dips down to below $10^{8} \mathrm{GeV}$ for a small range of values of the ratio $v_{2} / v_{1}$. This is a viable axion string model, both in the sense that it has no domain wall problem and that it is in no danger of violating even the most severe limits from the energy density in axions radiated from strings, which are dogged by theoretical uncertainty [19.18].

\section{3. "DEVIANT" AXION MODELS}

In this section we report of some forthcoming work [16] in which we attempt to give the lefthanded quarks a variety of $\mathrm{PQ}$ charges. A minimal departure from the DFSZ model is to have one or two quark doublets with PQ charge, and the other uncharged, which again defines six models. In the first three the 'special' doublets are either the $(u, d)_{L}$, or $(c, s)_{L}$, or $(t, b)_{L}$, labeled by I, IV, II, and in the last three ones either $(u, d)_{L}$ and
Figure 1. The lower bound on the PecceiQuinn symmetry-breaking scale $v_{a}$ for variant axion models described in the text and the DFSZ model, plotted as a function of $\beta$. Here, $\tan \beta=$ $v_{2} / v_{1}$, where $v_{1}$ and $v_{2}$ are the vacuum expectation values of the two higgs fields giving masses to the fermions. The labels indicate which of the right-handed quarks have Peccei-Quinn charge.

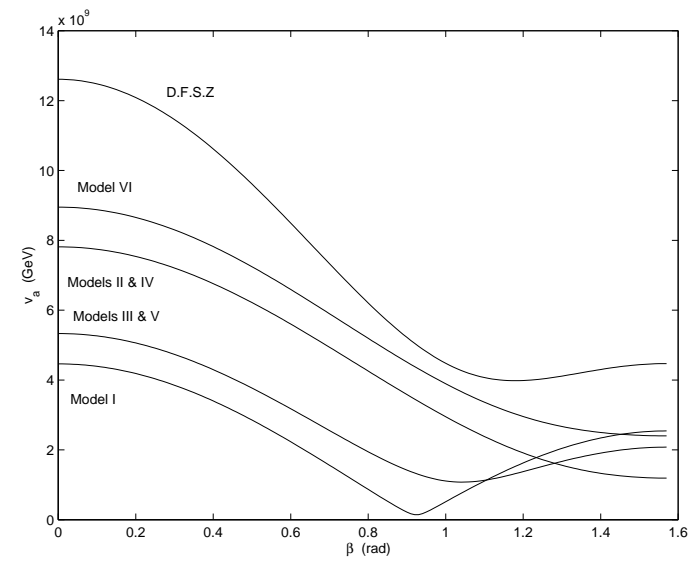

$(c, s)_{L}$, or $(u, d)_{L}$ and $(t, b)_{L}$, or $(c, s)_{L}$ and $(t, b)_{L}$ respectively, labeled by V, III, VI. We also have to assign PQ charges to the right-handed quarks. There are certain restrictions, as it turns out that if we try to introduce too much variety there are too many zeros in the Yukawa coupling matrices, and we cannot reproduce the observed structure of the Cabibbo-Kobayashi-Maskawa matrix [17]. One of the few realistic choices is to take the righthanded $u$-type quarks to have equal and opposite PQ charge to the left-handed quarks, and to give the right-handed $d$-type quarks PQ charge zero.

In order to do this we need at least three Higgs doublets $\phi_{n}$ and one singlet $\phi$. If we allow four Higgs doublets, we have the possibility of making the model supersymmetric, as discussed below. The general structure of the Yukawa couplings is

$\mathcal{L}_{Y}=f_{i j}^{n_{u}}\left(\bar{q}_{L i}^{\prime} \phi_{n_{u}} u_{R j}^{\prime}\right)+f_{i j}^{n_{d}}\left(\bar{q}_{L i}^{\prime} \phi_{n_{d}} d_{R j}^{\prime}\right)+h . c(3)$

where $n_{u}=1,3, n_{d}=2,4$ and $i, j=1,2,3$ are flavour indices. We could of course use $\tilde{\phi}_{3}=$ $i \sigma_{2} \phi_{3}^{*}$ instead of $\phi_{4}$, but this would be forbid- 
den in a supersymmetric model, as the superpotential (which determined the structure of the Yukawa terms) must be a function of $\phi_{n}$ and not $\tilde{\phi}_{n}$. The most general transformation laws giving an abelian symmetry are

$$
\begin{aligned}
u_{R i}^{\prime} & \longrightarrow e^{i \alpha T_{i j}^{u}} u_{R j}^{\prime} \\
d_{R i}^{\prime} & \longrightarrow e^{i \alpha T_{i j}^{d}} d_{R j}^{\prime} \\
q_{L i}^{\prime} & \longrightarrow e^{i \alpha T_{i j}} q_{L j}^{\prime} \\
\phi_{n} & \longrightarrow e^{i Q_{n} \alpha} \phi_{n}, \quad n=1,2,3,4 .
\end{aligned}
$$

In all our models, $Q_{1}=Q_{2}=1$, and $Q_{3}=Q_{4}=$ 0 . Let us consider Model I, where the transformation matrices are given by

$$
\begin{aligned}
T_{i j} & =\left(\begin{array}{lll}
1 & 0 & 0 \\
0 & 0 & 0 \\
0 & 0 & 0
\end{array}\right), \\
T_{i j}^{u} & =\left(\begin{array}{ccc}
-1 & 0 & 0 \\
0 & 0 & 0 \\
0 & 0 & 0
\end{array}\right), \\
T_{i j}^{d} & =0 .
\end{aligned}
$$

The implementation of these symmetries fixes $f_{i j}^{n}$ to have zeros in certain entries. The only nonzero entry in $f_{i j}^{1}$ is $f_{11}^{1}$, and we must also have $f_{i 1}^{3}=f_{1 j}^{3}=0$. For the $d$-type Yukawa couplings, we need $f_{2 j}^{2}=f_{3 j}^{2}=0$, and $f_{1 j}^{4}=0$. (Recall that it is $\phi_{1}$ and $\phi_{2}$ which have non-zero PQ charge). More details can be found in 16, 17.

Our model has tree-level FCNCs, and we must calculate the couplings in order to evaluate the constraints on the axion scale. In the flavour basis the relevant term of the QCD lagrangian is

$$
\begin{aligned}
\mathcal{L}_{i n t}= & -\frac{\partial^{\mu} a^{\prime}}{2 v_{a}}\left[\overline { u } _ { i } ^ { \prime } \gamma _ { \mu } \left(\left(1-\gamma_{5}\right) T_{i j}\right.\right. \\
& \left.+\left(1+\gamma_{5}\right) T_{i j}^{u}\right) u_{j}^{\prime} \\
& \left.+\bar{d}_{i}^{\prime} \gamma_{\mu}\left(1-\gamma_{5}\right) T_{i j} d_{j}^{\prime}\right]
\end{aligned}
$$

The quark mass matrices are diagonalised by the transformations

$$
\begin{aligned}
u_{R i}^{\prime}=U_{i j}^{R} u_{R j}, & d_{R i}^{\prime}=D_{i j}^{R} d_{R j}, \\
u_{L i}^{\prime}=U_{i j}^{L} u_{L j}, & d_{L i}^{\prime}=D_{i j}^{L} d_{L j} .
\end{aligned}
$$

Applying these transformations to (6) and going to the mass basis, the lagrangian takes the form

$$
\mathcal{L}_{i n t}=\frac{\partial^{\mu} a^{\prime}}{2 v_{a}}\left[2 \bar{u}_{i} \gamma_{\mu} \gamma_{5} S_{i j}^{u} u_{j}-\bar{d}_{i} \gamma_{\mu}\left(1-\gamma_{5}\right) S_{i j}^{d} d_{j}\right](6)
$$

where $S^{u}=U^{L^{\dagger}} T U^{L}$ and $S^{d}=D^{L^{\dagger}} T D^{L}$. By definition, the Cabbibo-Kobayashi-Maskawa matrix is $V_{C K M}=U^{L \dagger} D^{L}$, so it is obvious that

$S^{d}=V_{C K M}^{\dagger} S^{u} V_{C K M}$.

Thus FCNCs are generally present, in both vector and axial-vector currents in the $d$-type quark sector. Now, it is clear from the structure of the $u$-type Yukawa couplings that $U_{L}$ and $U_{R}$ have a block diagonal structure, with zeros in the first row and first column, and thus that $S^{u}=T$. Hence one can easily work out $S^{d}$ from knowledge of the elements of the CKM matrix.

For the process $K^{+} \rightarrow \pi^{+} a$ the interesting part of the interaction lagrangian (6) is the one giving the transition of $s \rightarrow d$ quarks. In this case

$\mathcal{L}_{\text {int }}=-\frac{\partial^{\mu} a^{\prime}}{2 v_{a}}\left[\bar{s} \gamma_{\mu}\left(g_{s d}^{V}+g_{s d}^{A} \gamma_{5}\right) d+\right.$ h.c. $]$

where $g_{s d}^{V}$ and $g_{s d}^{A}$ are introduced, being the vector and axial vector parts of the asd coupling. It is only the vectorial coupling which contributes to $K^{+} \rightarrow \pi^{+} a$, which is

$g_{s d}^{V}=V_{u d}^{*} V_{u s}$

where $\left|V_{u d}\right| \approx 0.98$ and $\left|V_{u s}\right| \approx 0.22$.

The relevant experimental limit is $\operatorname{Br}\left(K^{+} \rightarrow\right.$ $\left.\pi^{+} a\right)<3.0 \times 10^{-10}$ (at $90 \%$ confidence) [20]. This leads to a lower bound on the axion energy scale 16

$v_{a}>1.7 \times 10^{11} \times g_{d s}^{V} \quad \mathrm{GeV}$.

Thus for Model I, $v_{a}>3.7 \times 10^{10} \mathrm{GeV}$. The limits for all models, analagously derived, are listed in Table 2, taken from 16]. With the exception of Model III, these bounds are all stronger than the corresponding supernova bound [16]. The bound on Model III is weak because of the smallness of $V_{t d}$ and $V_{t s}$.

A theory with four Higgs doublets and a Higgs singlet has an additional three massive pseudoscalars. All of them can in principle mediate neutral meson mixing. $B \bar{B}$ is a particularly strong constraint, which forces the masses of the pseudoscalars to be greater than about $10^{6} \mathrm{GeV}$, [16] unless they are for some reason very weakly coupled to the $b$. Making a massive pseudoscalar 
Table 2

Limits on the axion scale $v_{a}$ from the flavourchanging process $K^{+} \rightarrow \pi^{+} a$.

\begin{tabular}{lll}
\hline Model & $\begin{array}{l}\text { Charged } \\
\text { doublets }\end{array}$ & $\begin{array}{l}\text { Limit } \\
(\mathrm{GeV})\end{array}$ \\
\hline I & $u d$ & $3.7 \cdot 10^{10}$ \\
II & $t b$ & $6.1 \cdot 10^{7}$ \\
III & $u d, t b$ & $3.7 \cdot 10^{10}$ \\
IV & $c s$ & $3.6 \cdot 10^{10}$ \\
V & $c s, u d$ & $7.3 \cdot 10^{10}$ \\
VI & $c s, t b$ & $3.6 \cdot 10^{10}$ \\
\hline
\end{tabular}

is not a problem in principle, as there are in general terms such as $\lambda \phi^{2} \phi_{1}^{T} \phi_{2}$, which contribute pieces of order $\lambda v^{2}$ to the masses. However, this leads to some kind of fine tuning in the Higgs potential, an ugly feature of these models which seems unavoidable.

One of the lessons we learn from these studies is that there is plenty of freedom in assigning the Peccei-Quinn symmetry properties of the quarks, particularly in the right-handed sector. If we wish to assign different charges in the left-handed sector, we must pay the price in inducing flavourchanging neutral currents mediated by the extra pseudoscalar bosons that are a result of the extra Higgs fields in such models. To keep these at an acceptable level we must tune the Higgs potential such that these pseudoscalars have masses greater than about $10^{6} \mathrm{GeV}$, while keeping the electroweak Higgs vacuum expectation value at $246 \mathrm{GeV}$.

M.H. is supported by PPARC Advanced Fellowship B/93/AF/1642 and by PPARC grants GR/L56305 and GR/L55759.

\section{REFERENCES}

1. R.D. Peccei and H.R. Quinn, Phys. Rev. Lett. 38 (1977) 1440; R.D. Peccei and H.R. Quinn, Phys. Rev. D16 (1977) 1791.

2. S. Weinberg, Phys. Rev. Lett. 40 (1978) 223; F. Wilczek, Phys. Rev. Lett. 40 (1978) 279.

3. J.E. Kim, Phys. Rev. Lett. 43 (1979) 103; M.A. Shifman, V.I. Vainstein and V.I. Zakharov, Nucl. Phys. B166 (1980) 4933.
4. A.P. Zhitnitskii, Sov. J. Nucl. Phys. 31 (1980) 260; M. Dine, W. Fischler and M. Srednicki, Phys. Lett. B104 (1981) 199.

5. R.D. Peccei, T.T. $\mathrm{Wu}$ and T. Yanagida, Phys. Lett. B172 (1986) 435.

6. L.M. Krauss and F. Wilczek, Phys. Lett. B173 (1986) 189.

7. J.E. Kim, Phys. Rep. 150 (1987) 1; H.-Y. Cheng, Phys. Rep. 158 (1988) 1; M.S. Turner, Phys. Rep. 197 (1990) 67; G.G. Raffelt, Phys. Rep. 198 (1990) 1.

8. G.G. Raffelt and D. Seckel, Phys. Rev. Lett. 60 (1988) 1793; M.S. Turner, Phys. Rev. Lett. 60 (1988) 1797; R. Mayle et al., Phys. Lett. B203 (1988) 188; R. Mayle et al., Phys. Lett. B219 (1989) 515; A. Burrows, M.S. Turner and R.P. Brinkmann, Phys. Rev. D39 (1989) 1020.

9. W. Keil et al., Phys. Rev. D56 (1997) 2419.

10. C.Q. Geng and J.N. Ng, Phys. Rev. D39 (1989) 1449.

11. S.L. Cheng, C.Q. Geng and W.-T. Ni, Phys. Rev. D52 (1995) 3132.

12. P. Sikivie, Phys. Rev. Lett. 48 (1982) 1156.

13. A. Vilenkin and E.P.S. Shellard, Cosmic Strings and Other Topological Defects, Cambridge University Press, Cambridge, 1994.

14. M. Hindmarsh and P. Moulatsiotis, Phys. Rev. D56 (1997) 8074.

15. J.L. Feng et al., Phys. Rev. D57 (1998) 5875.

16. M.B. Hindmarsh and P. Moulatsiotis, in preparation.

17. M.B. Hindmarsh and P. Moulatsiotis, in preparation.

18. D. Harari and P. Sikivie, Phys. Lett. B195 (1987) 361; C. Hagmann and P. Sikivie, Nucl. Phys. B363 (1991) 247.

19. R. Davis, Phys. Rev. D32 (1985) 3172; A. Vilenkin and T. Vachaspati, Phys. Rev. D35 (1987) 1138; R.A. Battye and E.P.S. Shellard, Phys. Rev. Lett. 73 (1994) 2954, ibid 76 (1996) 2203; R.A. Battye and E.P.S. Shellard, Critique of the Sources of Dark Matter in the Universe, Proc. 1994 Int. Symposium at UCLA.

20. E787 Collaboration, S. Adler at al., Phys. Rev. Lett. 79 (1997) 2204. 